\title{
Reactions of Vinylcyclopropane and Bicyclopropyl Compounds With Maleic Anhydride
}

\author{
C. K. Jankowski, ${ }^{* 1}$ A. Pelletier, ${ }^{1}$ Eduardo Díaz, ${ }^{2}$ Jacqueline M. R. Belanger, ${ }^{3}$ J.R. J. Paré, ${ }^{3}$ C. \\ Lamouroux, ${ }^{1}$ and J. Boivin ${ }^{4}$ \\ ${ }^{1}$ Département de Chimie et Biochimie, Université de Moncton, Moncton, NB, E1A 3E9, Canada \\ 2 Instituto de Química, Universidad Nacional Autónoma de México. Circuito Exterior, Ciudad Universitaria. \\ Coyoacán 04510, México, D.F. \\ 3 CTE, Division of green chemistry, Environment Canada, Ottawa, 325 River Road, K1A 0H3 Canada \\ ${ }^{4}$ Institut des substances naturelles, CNRS, 91198 Gif- sur- Yvette, France. Tel 1506858 4331, fax 4541 \\ jankowc@moncton.ca
}

Received June 22, 2009; accepted November 20, 2009

\begin{abstract}
Vinylcyclopropane and bicyclopropyl $\mathrm{C}_{6}$ compounds are studied as diene precursors in the Diels-Alder reaction. Their rearrangement under different thermal and microwave conditions leads to specific isomeric hexa-, penta- or butadienes, condensed with maleic anhydride as a dienophile. Adduct stereochemistries were compared to those of previously-synthesized model compounds, and are fully characterized with 2D NMR and GC-MS spectroscopies. Some unsaturated terpenes bearing vinylcyclopropane moieties were also condensed, and their adduct structures assigned.

Key words: Microwave, Thermal Diels-Alder Reactions, Diene Precursors, Vinylcyclopropane as a Diene
\end{abstract}

\section{Introduction}

One of the most useful reactions for building alicyclic structures is the Diels-Alder condensation (DA). Its scope and limitations, its different modes of operation, its control of adduct stereochemistry, and its mechanisms have all been widely studied, and several systematic rules have been discovered [1-3]. Several thermal, photochemical, catalytic, enzymatic or microwave (MW)-assisted, high-pressure and variable-temperature DA condensations were attempted (not always successfully) on the synthetic or biological building blocks, both dienes and dienophiles $[4,5]$.

The search for some diene analogs led to the design of more functional diene precursors, for example, vinyl-oxiranes, -aziridines or 1,3-diepoxides [6], or precursors. In this last case, the closest double bond alternative is the cyclopropane $[7,8]$. In "conjugation" to the vinyl or allylic bonds, this ensemble - when built on, for example, a six-carbon chain with the three carbons necessary for cyclopropane formation - will offer a hexadiene-like system.

As an extension of the vinylcyclopropane (1) systems based on bicyclopropyl (2), allyl cyclopropane (3) or cyclopropyl propenes (4) - all of six carbons - could also be considered as potential diene sources. Of course, in order to react as a 1,3-conjugated diene, the cyclopropane moiety (or moieties) of the vinylcyclopropane or bicyclopropyl derivatives should undergo an opening and numerous rearrangements [9-15].
Resumen. Vinilciclopropano y compuestos biciclopropilos $\mathrm{C}_{6}$ se estudiaron como precursores de dienos en reacciones de Diels-Alder. La transposición de estos compuestos bajo condiciones térmicas o de irradiación de microondas conduce específicamente a hexa-, penta- o butadienos isoméricos, los cuales cicloadicionan con anhídrido maleico como dienófilo. La estereoquímica de los aductos se estableció por comparación con aductos modelo previamente preparados y se caracterizaron por espectroscopia de RMN de dos dimensiones y CGEM. Algunos terpenos insaturados llevando consigo el sistema vinilciclopropano también se condensaron, y fue asignada la estructura de sus aductos.

Palabras clave: Microondas, reacciones térmicas de Diels-Alder, precursores de dienos, vinilciclopropanos como dienos.

The dienes obtained as a result of previously-referenced rearrangements of cyclopropane moieties of $\mathbf{1}$ or $\mathbf{2}$, could be identified as Diels-Alder adducts with maleic anhydride, which is a high-efficiency dienophile and compared to some model adducts obtained from the pure dienes (Scheme 1)

Although Diels-Alder reactions are feasible under a wide variety of conditions, we chose to perform thermal and MWassisted experiments because of their simplicity, and because of the proven "green" character of the latter. It is also of some interest to study the potential subtle differences between these two modes of activation of reagents entering cycloaddition, as has already been observed in our previous works (14).

It is expected that the reactions involving cyclopropane moieties will first lead to the production of conjugated six-

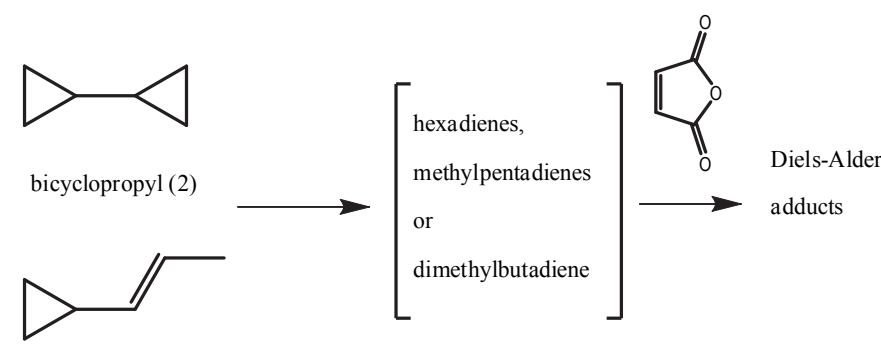

1-cyclopropyl propene (4)

Scheme 1 
carbon diene systems $\left(\mathrm{C}_{6}\right)$ with hexa, penta or buta- diene structures; these dienes react with maleic anhydride as per the stereochemistry revealed by the rearrangements [15]. Although some of these dienes do not lead directly to the s-cis-1,3-diene system necessary for efficient Diels-Alder addition the minor differences on the geometry of the dienes formed will be reflected by different adducts [16]. Because of the evident multistep character of the proposed synthesis, the stereochemistry of adducts produced from vinylcyclopropane cannot be predicted by Woodward-Hoffmann rules (2), however the dienes, once formed, should react with dienophile accordingly.

In this study we present the Diels-Alder reaction on the following compounds: allyl cyclopropane (3), 3-cyclopropyl 2-propene (4 cis and 4 trans), and 1-cyclopropyl propanol (5) as a compound leading via the dehydration of the previous two compounds, and the only two cyclopropane rings compound , bicylopropyl (2). In the series of terpenes which contain vinyl and cyclopropyl moieties, we performed the reactions on 2carene (6), and two model terpene dienes - terpinene (7) and $\alpha$-phellandrene (8) [17].

It is worth noting that terpenes were already used as diene precursors by Diels in 1938 for carene and thujene systems and even extended to cyclobutane-containing terpenes [18, 19]. It is also necessary to point out that 2-carene (6) has an endocyclic double bond and an exocyclic cyclopropane within its bicyclo $[4,1,0]$ heptane skeleton. This last can either undergo rearrangement to a bicyclo[2,1,2] heptane skeleton, or open its cyclopropane bond, both of which give the seven-member ring (18) (Scheme 2). This work is also an extension of our previous observations on cubebene (10), a tricyclic sesquiterpene extracted from Solidago Canadiensis species. Cubebene contains the vinylcyclopropane system and undergoes both diene formation and epimerisation under Micro Wave-assisted conditions. Finally; in this work we try to rationalise some of those results by studying simpler diene-generating systems $[14,21]$.

In order to have several models of maleic anhydride adducts for trans,trans-hexadiene (11 tt), trans, cis-hexadiene (11 ct), 4-methyl 1,3-pentadiene (12) and 2,3-dimethyl 1,3-butadiene (13) adducts from pure isomeric six-carbon dienes were synthesized, a series of terpenes ( 7 and $\mathbf{8}$ and their maleic anhydride adducts) were also prepared (Scheme 2, 3 and 5).

All Diels-Alder reactions were performed in two parallel ways: thermal and MW-assisted manner for all simple diene precursors and terpene compounds. The starting dienes were also subjected to attempts at thermal and MW-assisted isomerisation without maleic anhydride in order to evaluate their possible structural alteration prior to their reaction as dienic compounds.

It must be noted that Micro Wave-assisted treatments are based upon conditions that differ from those that prevail under conventional thermal reactions. The main difference is that the susceptibility of the reagents towards microwaves may well be very different from those of the products. Hence, the
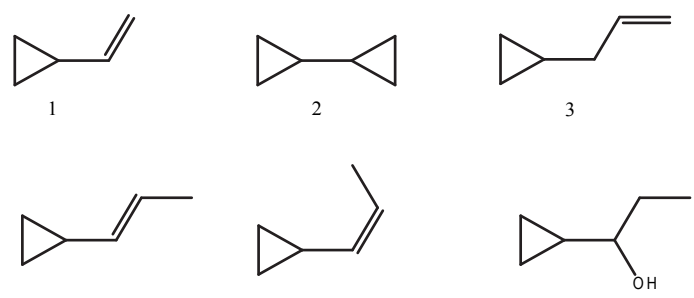

$4 \mathrm{t}$

$4 \mathrm{c}$
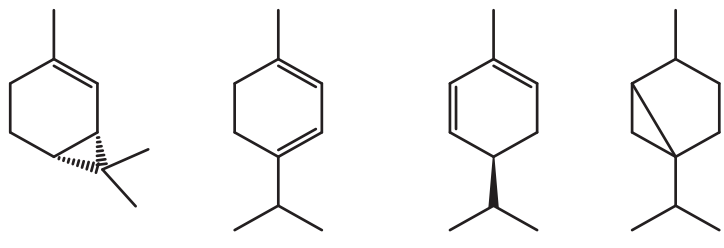

6

7

8

9
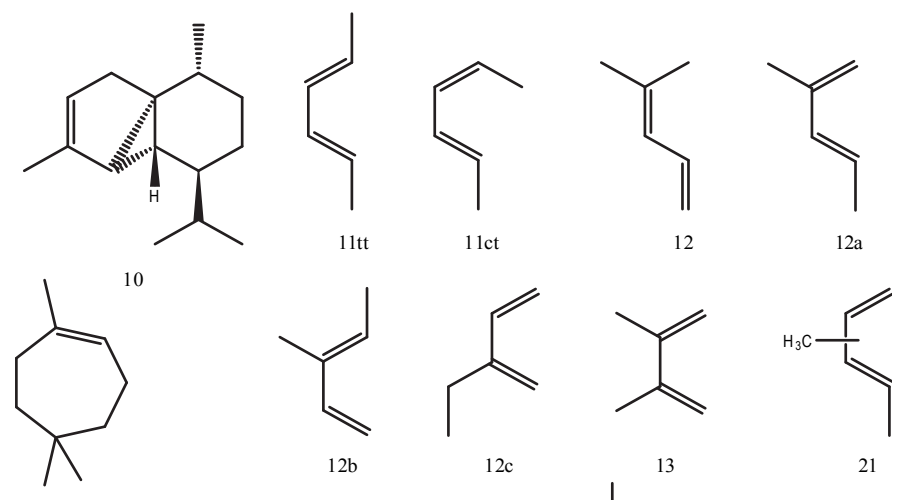

$12 \mathrm{~b}$

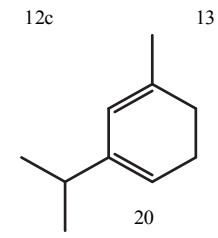

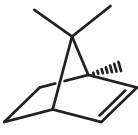

interaction between microwaves and the various components is selective with a bias toward compounds characterized by a higher dielectric constant. While this may offer advantages in terms of conversion rate (yields), it has significant effect on the speed of the reaction and the selectivity (less side reactions or reduced degradation). In our examples, this behaviour can be explained easily from the reduced interactions the products have towards microwaves (reduced value of dielectric constant). This not only implies that less energy is imparted to them (thus, polymerization is reduced), but also that reaction kinetics are increased: for a given applied power, assuming constant reflection, the electrical field increases over time. Electrical field intensity is well known to be the most important factor in Micro Wave-assisted processes such as chemical synthesis [22-24].

In all cases, adducts and some diene structures were modelled in order to evaluate stability while the $500 \mathrm{MHz}$ NMR studies will allow for unambiguous identification of single adducts or mixtures thereof. 


\section{Results and discussion}

The diene-generating systems from different vinylcyclopropanes were of two origins: simple systems containing one or two cyclopropane moieties (all of which were six-carbon compounds leading to dienes), and terpene-originating systems, such as 6,7 or 8 . The results of their condensation with maleic anhydride are presented in two following sections. (Scheme 3 and 5)

\section{Simple diene series}

In this series of experiments, four diene precursors were synthesized. In a mono cyclopropyl series - allyl cyclopropane (3) (alternative name 3-cyclopropyl 1-propene), 1-cyclopropyl propene (4 cis or 4 trans) and as a model to previous one 1cyclopropyl propanol (5) were synthesized. For the two cyclopropyl-containing series, the parent bicyclopropyl (2) was also prepared. 28):

These syntheses were achieved by several methods (24-

a. direct Grignard coupling reaction, by the WurtzGrignard approach with allyl bromide to obtain the allyl cyclopropane (3), as well as by the similar reaction on dicyclopropyl Zn (25);

b. step-by-step Grignard reaction of cyclopropylmagnesium bromide on propionaldehyde to obtain, isolate, and separate 1-cyclopropyl propanol (5) which can lead to cis (4 cis) or trans (4 trans) 1-cyclopropyl propene by dehydration, used as obtained, in a mixture condensed with maleic anhydride or as a pure $\mathbf{4}$ trans isomer separated from this mixture;

c. cyclopropylmagnesium bromide coupling to cyclopropyl bromide, leading to the bicyclopropyl (2);

d. in situ synthesis of olefins from 1-cyclopropyl propanol (5), later leading to dienes as in point $b$.

Details of these syntheses are summarized in the Experimental section.

These cyclopropane compounds (with the exception of carbinol 5) are unstable, and difficult to synthesize. The use of closed vessels (stainless steel reactor for thermal, volatile and all-glass tube for Micro Wave) helped reduce the loss of material, and allowed the transformation of most of the diene into adducts ( the procedures are described in the Experimental ).

As mentioned previously, the dienes formed from these precursors were reacted with the maleic anhydride. The resulting adduct mixtures made via both thermal and MW-assisted routes were characterized, especially with help of their GC retention time, Rt (Table 1 and Experimental), GC-MS and high-resolution 1D and 2D NMR spectroscopic data obtained for four independently-synthesized adducts made from highpurity dienes. Particular attention was given to the proton high-resolution NMR, and minor differences in mass spectra of adducts (Table 2). All model diene adducts (14-17) (Scheme
Table 1. Thermal and micro-wave assisted reaction on cyclopropane substrates

\begin{tabular}{|c|c|c|}
\hline Substrate & $\begin{array}{l}\text { Overall adducts } \\
\text { yield (\%) } \\
\text { Thermal / M-W }\end{array}$ & $\begin{array}{l}\text { Isomeric adducts obtained } \\
\text { (ratio)* }\end{array}$ \\
\hline 3 & $37-39 / 35$ & $\begin{array}{l}\text { - Hexadiene adducts } \\
83 \%, \mathbf{1 4} \text { and } \mathbf{1 5} \text { ratio 9:1 } \\
\text { (thermal) and 4:1 (M-W) }\end{array}$ \\
\hline $\begin{array}{l}\mathbf{4 t} \text { and } \mathbf{4 c} \text { (mixture } \\
\text { of trans and cis } \\
\text { isomers } 3: 1 \text { ) }\end{array}$ & $55 / 25$ & $\begin{array}{l}\text { - Hexadiene adducts } \\
\text { 90\%, } \mathbf{1 4} \text { and } \mathbf{1 5} \text { ratio 95:5 } \\
\text { (thermal) and 94:6 (M-W) } \\
\text { - Adduct } \mathbf{1 6} \text { (3\%) ( } \mathrm{M}-\mathrm{W} \\
\text { and thermal methods), } \\
\text { other methyl pentadiene } \\
\text { isomeric adducts : } 7 \%\end{array}$ \\
\hline $\mathbf{4 t}$ (pure) & $45 / 20(\max )$ & $\begin{array}{l}\text { - Adducts } \mathbf{1 4} \text { ( } 45 \%) \\
\text { and } \mathbf{1 5}(23 \%) \text {, methyl } \\
\text { pentadiene adducts } 36 \% \\
\text { (thermal) } \\
\text { - Adducts } \mathbf{1 4} \text { and } \mathbf{1 5} \text { ( } \\
30-35 \% \text { ratio } 4: 1) \text { methyl } \\
\text { pentadiene adducts ( } 20 \text { - } \\
25 \%)(\mathrm{M}-\mathrm{W})\end{array}$ \\
\hline 5 & $24 / 13$ & $\begin{array}{l}\text { - Adducts } 14 \text { 45\%, } 15 \\
23 \% \text { (thermal and M-W) } \\
\text { - Isomeric pentadiene } \\
\text { adducts up to } 17 \%\end{array}$ \\
\hline 2 & $20-25 / 46$ & $\begin{array}{l}\text { Adducts } \mathbf{1 4} 65 \%, \mathbf{1 5} 23 \% \text {, } \\
\text { methyl pentadienes } 10 \% \text {, } \\
\mathbf{1 7} \text { (traces), (thermal) } \\
\text { Adducts } \mathbf{1 4} 50 \%, \mathbf{1 5} 23 \text { - } \\
25 \% \text {, isomeric pentadiene } \\
\text { adducts } 15 \%, 165 \%, 17 \\
3 \%\end{array}$ \\
\hline
\end{tabular}

* ratio of Diels-Alder adducts evaluated from m/z 180 ion mass chromatogram

3) displayed the isomass molecular ion at $\mathrm{m} / \mathrm{z} 180$, with a major fragmentation pattern leading to fragments at $\mathrm{m} / \mathrm{z} 152$, and retro-Diels-Alder fragments of various intensities at $\mathrm{m} / \mathrm{z}$ 98, 70 and 82 (Table 2).

In order to position the given adducts, their Rt and the GC-MS single-ion monitoring at m/z 180 were used (Table 2). In principle, the hexadiene, methyl pentadiene and dimethyl butadiene adducts - formed from 11 tt, $11 \mathrm{ct}, \mathbf{1 2}$ and $\mathbf{1 3}$ dienes - are identified according to those parameters and confirmed by NMR.

From these results it was observed that, for a thermal reaction starting with allyl cyclopropane (3), two maleic anhydride adducts were observed, $\mathbf{1 4}$ and $\mathbf{1 5}$ (ratio 9:1), corresponding to 

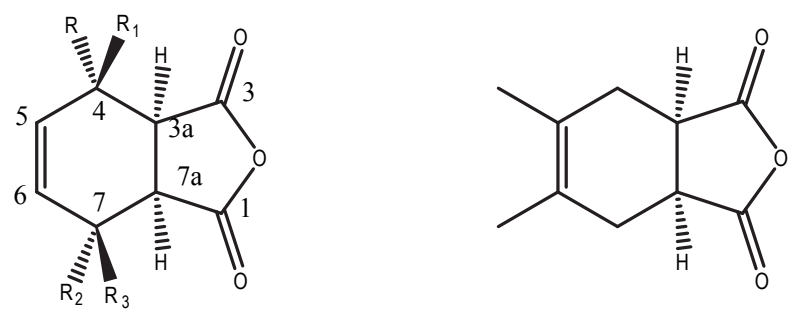

$\mathrm{R}=\mathrm{R}_{2}=\mathrm{CH}_{3}$

$$
\mathrm{R}_{1}=\mathrm{R}_{3}=\mathrm{H}
$$

$\mathrm{R}_{1}=\mathrm{R}_{2}=\mathrm{H}$

$\mathrm{R}=\mathrm{R}_{1}=\mathrm{H}$

$\mathrm{R}_{2}=\mathrm{R}_{3}=\mathrm{CH}_{3}$

17

Scheme 3

trans,trans-2,4-hexadiene (14) (major) and cis,trans-2,4-hexadiene (15) (minor) adducts. The dienes were formed first by the isomerisation of the double bond from 1,2 - to 2,3 - position of the diene, and by the cyclopropane opening. As witnessed by the formation of the same adducts from compounds $\mathbf{4 t}$ and $\mathbf{4 c}$.

The alternative way - first the opening of the cyclopropane followed by the isomerisation of the double bond system to the conjugated diene could also be considered. The dienes $11 \mathrm{tt}$ and 11ct are both formed from 3 but product 16 cannot be formed (as it is from $\mathbf{4 t}$ alone or from the mixture of $\mathbf{4 t}$ and 4c). This alternative way was proposed by the Referee of our paper. It cannot however explain the diene 12a which gives the adduct $\mathbf{1 6}$ as shown in Scheme 4.

In a MW-assisted reaction the same yield (ca 35\%) and $4: 1$ ratio of adducts were observed, however, less secondary products were formed.

For the cis and trans 1:3 mixture of compound 4, the thermal reaction gives a much higher yield of adducts than does the MW-assisted reaction (55\% to $25 \%$ ): the major adduct 14 comes from trans, trans-2,4-diene (11 tt) adduct, and the minor adduct $\mathbf{1 5}$ comes from the cis,trans diene (11 ct) (ratio 95:5 in thermal and 94:6 in MW method). The reaction was also repeated for the purified $\mathbf{4} \mathbf{t}$ olefin, with results as described in Table 2.

Table 2. Thermal and micro-wave $(\mathrm{M}-\mathrm{W})$ assisted reactions of model dienes and maleic anhydride

\begin{tabular}{ccccc}
\hline \multirow{2}{*}{ Diene } & Adduct obtained & \multicolumn{2}{c}{ Yield (\%) } & \multirow{2}{*}{$\mathrm{R}_{\mathrm{t}}^{* *}$} \\
\cline { 3 - 4 } & & Thermal & Micro-wave \\
\hline $\mathbf{1 1 t t}$ & $\mathbf{1 4}$ & 95 & 90 & 1.00 \\
$\mathbf{1 1 c t}$ & $\mathbf{1 5}$ & 69 & 60 & $0.95-0.97$ \\
$\mathbf{1 2}$ & $\mathbf{1 6}$ & 43 & - & 0.82 \\
$\mathbf{1 3}$ & $\mathbf{1 7}$ & 34 & 52 & 0.71 \\
\hline
\end{tabular}

**relative retention time on column HP-5MS $60 \mathrm{~m}$ of this adduct $7.30 \mathrm{~min}$ was normalized to 1.00
In this last case, the minor methyl pentadiene adducts were also identified - in particular, the adduct originating from the 4-methyl-1,3-pentadiene (12), identified in an MW-assisted reaction $(3 \%)$. This result could be related to the position of the double bond in "conjugation" to the cyclopropyl moiety, which plays an activation role for the opening of either of two cyclopropane bonds, thereby leading to the diene formation (Scheme 4).

In the next diene precursor studies, 1-cyclopropyl propanol (5) was subjected to Diels-Alder reaction with maleic anhydride, giving $24 \%$ of adducts in thermal reaction and less than $15 \%$ under MW heating. The major adduct 14 corresponded to the trans,trans -diene (45\%), followed by adduct $15(13 \%)$. The lower overall yield of these reactions is probably related to the dehydration of alcohol necessary to produce the first double bond. The elimination of water from such cyclopropyl carbinol was not catalyzed by, for example, base or acid addition in either set of reaction conditions used.

The last and most challenging reaction of the bicyclopropyl (2), formed from several thermal and microwave generally laborious attempts, lead to a conclusion that the yields of adducts are much higher for the micro-wave assisted closed

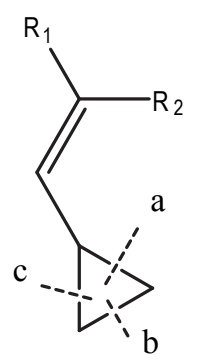

a or c : 2, 4-hexadienes

b : 2-methyl-1, 3-pentadiene

$\mathrm{R}_{1}$ or $\mathrm{R}_{2}: \mathrm{CH}_{3}$ or $\mathrm{H}$

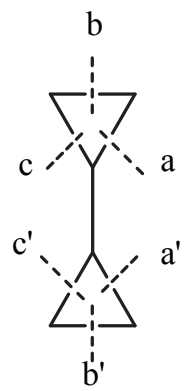

a, a' : 2, 4-hexadienes ( as well as via $\mathrm{a}, \mathrm{c}^{\prime} ; \mathrm{a}^{\prime}, \mathrm{c}$ or $\mathrm{c}, \mathrm{c}^{\prime}$ routes)

b, b' : 2, 3-dimethyl-1, 3-butadiene

a, b' : 2-methyl-1, 3-pentadiene (as well as c, b')

$\mathrm{a}$ is equivalent to $\mathrm{c}$ and $\mathrm{a}^{\prime}$ is equivalent to $c^{\prime}$ in this case

but
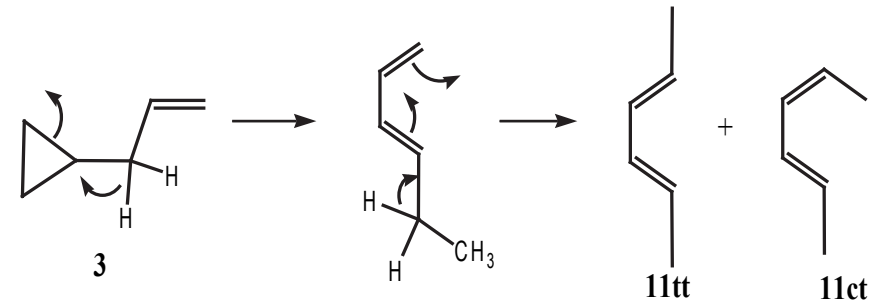

Scheme 4 
vessel method, $46 \%$ than for thermal $20-25 \%$, however even in the Micro Wave-assisted reaction, the presence of adduct 14 (50\% yield) was followed by 15 (23-25\%), 4-methyl 1,3-pentadiene adduct (16) (5\%), and finally 2,3-dimethyl-1,3-butadiene adduct (17) (1\%). In the thermal series, the ratio and yields of 14, 15 and methyl pentadiene adducts were similar, and butadiene adduct $\mathbf{1 7}$ was obtained at traces level only. These last results indicate that the single cyclopropane ring in bicyclopropyl (2) rearranged nonspecifically to two hexadienes by cleavage of a, a' (or a, c') cyclopropane bonds. It was observed also that the cleavage of cyclopropane bond followed either the already-observed cleavage of a, b' bond, or a new cleavage of $\mathrm{b}, \mathrm{b}$ ' bond, to give minor pentadiene and butadiene adducts, respectively (Scheme 4).

The most important factors in the identification of the adducts were the polynuclear 1D and 2D NMR spectroscopies. NMR analysis of these mixtures was performed using specific signals, with the signals from adduct methyls used as a starting point. For example, the distinction between the cis, trans and trans, trans 2,4-hexadiene adducts $\mathbf{1 4}$ and $\mathbf{1 5}$ of two methyl proton chemical shifts (1.38 and $1.46 \mathrm{ppm}$ ), protons 4 and 7 as well as their coupling patterns to the maleic bridge protons and NOE effects. For example, both bridge protons and $\mathrm{CH}_{3}$ as well as $\mathrm{CH}_{3}$ and vinylic protons $(\mathrm{H}-5, \mathrm{H}-6)$ showed strong NOE for adduct $\mathbf{1 4}$ but one strong NOE effect only between H-3a alpha and $\mathrm{CH}_{3}$ alpha and also $\mathrm{CH}_{3}-\mathrm{C}_{4}$ and $\mathrm{H}-5$ for adduct $\mathbf{1 5}$ was observed.

The results presented here led to the conclusion that, in all cases, there is an overwhelming propensity for the $\mathbf{1 1} \mathbf{t t}$ diene formation from cyclopropyl propene precursors, leading to the adduct 14.

The bicylopropyl (2) system showed the possibility of the rearrangement of the cyclopropane by several $\mathrm{C}-\mathrm{C}$ bond cleavages, leading to the formation of $\mathbf{1 1} \mathbf{~ t t}$, but also $\mathbf{1 1} \mathbf{~ c t}$, along with a methyl pentadiene fraction and traces of $\mathbf{1 3}$. It should also be mentioned that other remaining isomeric methyl pentadienes (12, 2-methyl, all 3-methyl etc.) adducts were not synthesized separately. However, for this last series, the yields of these reactions remain low - less than $20 \%$. For the $\mathrm{MW}$-assisted reactions the polymerisation is certainly lower; but at best, these yields are in the same general range as for thermal reactions.

The addition of maleic anhydride is efficient in the presence of aprotic and anhydrous solvents. When preparing adducts, it is useful to replace the benzene or toluene solvent with ethyl acetate, to help reduce the polymerisation and opening of the anhydride to form the diacid. In this manner we also avoided the spontaneous isomerisation of $\mathbf{1 1} \mathrm{ct}$ to $\mathbf{1 1} \mathbf{~ t t}$, observed for hexadienes under lengthy condensation (e.g. 24 hrs or longer).

\section{Terpene series}

In a series of more complex compounds which contain vinyl and cyclopropyl moieties, we performed the reactions on 2carene (6) with the help of two model terpene dienes, terpinene (7) and $\alpha$-phellandrene (8) (Scheme 5).

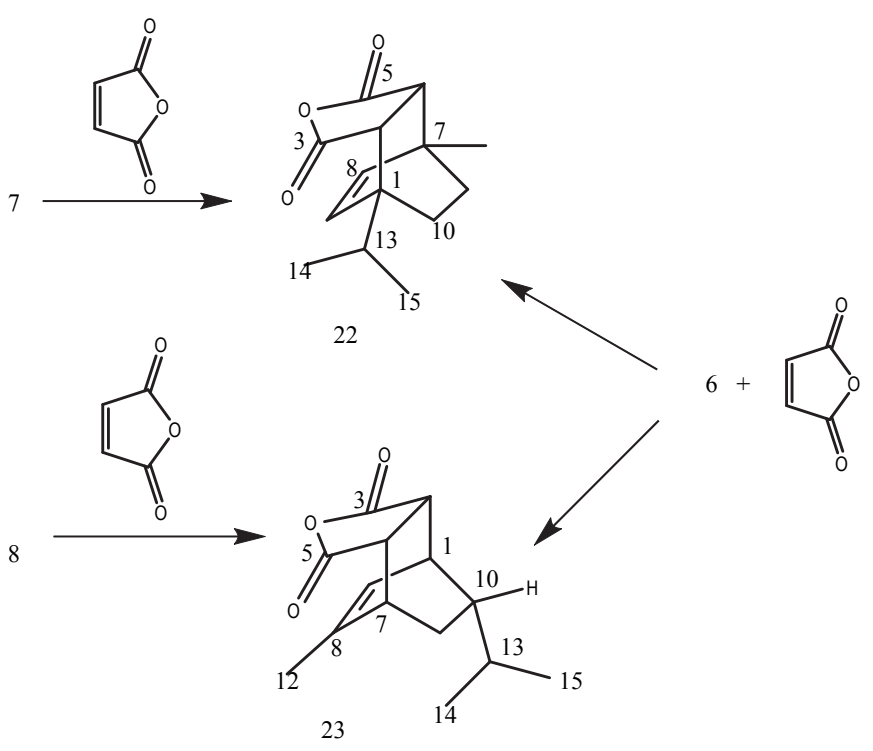

Scheme 5

The rearrangements of the 2-carene (6) should lead, via one of three possible cyclopropane bond cleavages, to either:

a. 1,7,7-trimethyl bicyclo $[2,1,2]$ hept-2-ene (19), a possible precursor to the $\alpha$-terpinene (7) and á-phellandrene (8) after the corresponding allylic proton shift, the cyclopropane bond opening corresponded to the favoured E(pro-trans)-cyclopropane bond cleavage;

b. the unobserved iso-terpene skeleton of 1-methyl 3-isopropyl 1,3-cyclohexadiene (20) corresponding to the cleavage of the homo allylic to the double bond cyclopropane bond; or

c. 1,5,5-trimethyl cycloheptene (18) skeleton terpenes corresponded to the unfavoured $Z$ (pro-cis)-cyclopropane bond cleavage (28). (Schemes 2 and 5).

The reaction of 2-carene (6) carried out under thermal or MW-assisted conditions with maleic anhydride led to a mixture of two adducts identified as the maleic anhydride of terpinene (7) and phellandrene (8) adducts, respectively; almost invariably the ratios were between 4:6 to $1: 2$ in favour of the phellandrene-originating product $\mathbf{2 3}$. The parallel synthesis of these two compounds from the addition of single-terpene dienes $\mathbf{7}$ and $\mathbf{8}$ to maleic anhydride allowed for their unambiguous identification. This result means that the 2-carene (6) is non-specifically rearranged to these two dienes, which react with the dienophile.

In order to further test this hypothesis, the 2-carene (6) is heated or irradiated under MW without maleic anhydride in several solvents (such as ethyl acetate, benzene or toluene); $\mathrm{GC}$ reveals that both individual terpene dienes are formed. All three terpenes show good stability under these conditions, less than $2 \%$ of 7 rearranges to 8 after $24 \mathrm{~h}$ of conventional heating or under MW irradiation in these solvents (18). 
The NMR spectrum from terpene adducts $\mathbf{2 2}$ and $\mathbf{2 3}$ (see Experimental) display some interesting features, as confirmed by $2 \mathrm{D}$ experiments. The most interesting coupling observed, which confirms the proposed structures for adduct $\mathbf{2 3}$, is the 4J coupling between the H-9 vinylic proton and the H-10 beta proton; this confirms the proposed alpha orientation of the isopropyl group. NOESY experiments on the same system confirm this adduct's structure by showing the strong effects between H-2 - H-10 exo and H-6 - H-11 exo as well as H-9 interaction with both isopropyl methyls. For adduct 22, however, the relevant features used for the confirmation of the structure were the dipolar correlation between $\mathrm{H}-2$ and $\mathrm{H}-10$ exo and $\mathrm{H}-6$ and $\mathrm{H}-11$ exo protons. As in the previous cases, the conformational energies for all terpene adducts were calculated, both for structures resulting from exo or endo approaches or having isopropyl group in equatorial position for adduct $\mathbf{2 2}$.

\section{Conclusion}

The aim of this study was to test the potential of cyclopropanebearing precurssors $\mathbf{2 - 5}$ to produce dienes via the rearrangements.

It was observed that they are able to produce Diels-Alder adducts in both thermal and MW-assisted reactions, once their rearrangements are completed. The formation of dienes from the strained cyclopropane precursors was relatively easy, as shown by the diene adducts obtained. The same cannot be said about the synthesis of all cyclopropane compounds in this study, which was laborious and difficult: products were unstable, and yields were low. The most suitable solvent for these reactions was found to be ethyl acetate, which was preferred over chloroform, benzene, toluene, and water.

Although the results of condensations under MW-assisted conditions generally lead to cleaner mixtures of products in less time and with less solvent used, the expected differences between condensations under thermal and MW-assisted modes were not observed. The same could be concluded about the reactions in general, except that the bicyclopropyl (3) under MW-assisted conditions produces more butadiene. It is necessary to point out that the MW-assisted reactions were performed via heating through the solvent mode; reactions using focused MW should be also performed, in order to verify this conclusion.

In general however for all cyclopropane precursors 2-5 the lack of more pronounced selectivity of the rearrangements toward some specific dienes, except hexadienes, was observed under both heatings conditions used. The Diels-Alder condensation and the ratio of diene adducts obtained in the sequence of reactions attempted is then only the indication of the selectivity of the multi-step rearrangement processus. This also may be concluded about terpenes with more rigid structures, where the stereochemistry of the starting vinylcyclopropane could lead to more specific adduct isomers. However, many questions of mechanism of the rearangment remain unanswered, for example of whether the vinylcyclopropane systems allow the 1,5-sigmatropic shifts through the cyclopropane ring, because of its partial double bond character, or whether the rearrangement could instead proceed through a series of 1,3sigmatropic and allylic shifts.

As a further perspective to this study, the use of the aforementioned focused MW and photochemical reactions on the substrates $\mathbf{2 - 5}$ is an interesting alternative. It will also be interesting to build (for example) cyclohexene structure from the vinylcyclobutane (for 6-carbon-containing compounds).

\section{Acknowledgements}

The authors are indebted to Nuclear Research Centre of Saclay (France) and to the Université de Moncton (Canada) for constant support of this research projects. This work was supported by the Youth Canada Environment Canada scholarship to AP. We would like to address special thanks to Dr. D. Lesage (UdeParis) and S. Arseneau (Atlantic Baptist University) for valuable technical assistance and to Referee 1 of our revised paper for comment on sequence of isomerisation-cyclopropane opening mechanism.

\section{Experimental}

\section{Materials and methods}

All cyclopropyl products and most of reagents were purchased from Aldrich Chemicals. The purification of volatile material was made using Buchi- Kugelrohr distillation apparatus and several flash chromatography on usual absorbents (Si-gel).

\section{GC- MS measurements}

GC/MSD Analysis: $10 \mu \mathrm{L}$ aliquot was pipetted from each sample and diluted with $1 \mathrm{~mL}$ of hexane then were analyzed on a GC/MSD system consisting of an Agilent 6890N GC equipped with an Agilent 7683 automatic liquid sampler and interfaced directly to an Agilent 5973N mass selective detector controlled via Agilent's ChemStation software. A 60-m HP-5MS capillary column $(0.25 \mathrm{~mm}$ id, $0.25 \mu \mathrm{m})$ was used. Elution conditions were as follows: constant flow of $1,0 \mathrm{~mL} / \mathrm{min}$ with an initial pressure of $18.54 \mathrm{psi}$; initial oven temperature of $70^{\circ} \mathrm{C}$ maintained for $2 \mathrm{~min}$, then heated at $20^{\circ} \mathrm{C} / \mathrm{min}$ up to $230{ }^{\circ} \mathrm{C}$ where it was maintained for $5 \mathrm{~min}$; injection volume of 0.2 $\mu \mathrm{L}$ in a split-less mode at $250{ }^{\circ} \mathrm{C}$ with a purge-off time of 2.00 min.; the MSD was operated in selected ion monitoring ( within $\mathrm{m} / \mathrm{z} 100-300$ range ) with a transfer line kept at $280^{\circ} \mathrm{C}$, quadrupole at $150{ }^{\circ} \mathrm{C}$ and source at $230^{\circ} \mathrm{C}$. Tuning was performed using the auto-tune feature with perfluorotributylamine (PFTBA) and the electron multiplier voltage was nominally set at $1529 \mathrm{~V}$.

The electron impact (EI) mass spectra are given in $\mathrm{m} / \mathrm{z}$ (I\%) of the most intense ion . 
NMR

NMR high resolution experiments were performed using Varian INOVA spectrometer, at $500 \mathrm{MHz}$ for proton and 125 $\mathrm{MHz}$ for carbon-13 respectively. In all cases the samples were prepared in $\mathrm{CDCl}_{3}$ (or in different solvents as indicated) as ca $5 \%$ solution at $25^{\circ} \mathrm{C}$, chemical shifts were referred to the TMS in $\delta$ scale, are expressed in ppm and the coupling constants in Hz: s (singlet), d (doublet), t (triplet), q (quadruplet), m (multiplet).

Selected 2D experiments were run using a standard Varian package of INOVA programs for COSY and NOEs. HSQC experiments for short range couplings, less than $140 \mathrm{~Hz}$, and one bond coupling were used. The long-range proton-carbon experiments (HMBC) were recorded with Varian's program.

Other spectrometers used for more routine analysis of known products were Bruker apparatus $200 \mathrm{MHz}, 300 \mathrm{MHz}$ and $400 \mathrm{MHz}$ NMR (UdeMoncton, CEA Saclay, IRSN CNRS Gif,respectively as indicated in the experimental ). The chemical shifts are expressed in $\ddot{a}$ (ppm) and coupling constants in Hz.

All the 1D and 2D detailed high resolution NMR spectra are available on request from the corresponding author (CKJ, Canada).

\section{Synthesis of substrates 2-5}

Allylcyclopropane (alternative name: 3-cyclopropyl 1-propene) (3)

Compound $\mathbf{3}$ was synthesized according to the modified procedure of Farneth (29) via Grignard-Wurtz coupling between the commercial cyclopropylmagnesium bromide and allyl bromide at room temperature. Yield of 3: $25-28 \%$, bp 55-60\% 25 torr (product should be kept under Nitrogen)

${ }^{1} \mathrm{H}$ NMR (200 MHz, $\mathrm{CDCl}_{3}$ ) ( $\delta$, ppm, \# of protons): 0.1$0.9(5 \mathrm{H}), 1.95(2 \mathrm{H}), 4.9(1 \mathrm{H}$, broad d, 10), $5.1(1 \mathrm{H}$, broad d, q, $15), 5.72(1 \mathrm{H}, \mathrm{tt})$

\section{1-cyclopropyl propanol (5)}

Carbinol 5 was synthesized according to modified procedure of Ferreri and Khusid $(28,30)$ from ethylmagnesium bromide and cyclopropenyl aldehyde at -20 to $-78^{\circ} \mathrm{C}$ in ether or in tetrahydrofurane, with moderate (56-70\%) yields. The synthesis was not optimised. When the dicyclopropyl zinc method is used with propionaldehyde as a starting aldehyde according to Reetz methodology (25), the yields of these reactions did not exceeded $25 \%$ and further improvements were abandoned because of the lack of variable temperature probe NMR facility and low temperature reactions incompatible with the microwave in use.

The analytical sample of carbinol, bp ca $135-140^{\circ} \mathrm{C}$ (dec.), was stocked under the dry nitrogen atmosphere.

${ }^{1} \mathrm{H}$ NMR $\left(\mathrm{CDCl}_{3}, 400 \mathrm{MHz}\right)$ : cyclopropyl signals 0.51 $(2 \mathrm{H}), 0.23(2 \mathrm{H}), 0.88-0.9(1 \mathrm{H}), 2.82(\mathrm{~m}, 1 \mathrm{H})$, chain signals $1.60(\mathrm{q}, 2 \mathrm{H}), 1.01(\mathrm{t}, 3 \mathrm{H})$;
${ }^{13}$ C NMR: 2.2, 2.7 and 10.2 (cyclopropyl), 17.5 (C3), 30.7 (C2), $75.2(\mathrm{C} 1)$; (23).

$$
\text { MS (EI, } m / z, \%): \mathrm{M}^{+} 100 \text { (2), } 99 \text { (M-1,100\%), } 71 \text { (34), } 57
$$

\section{Trans and cis 1-cyclopropyl propene (4t and 4t)}

The 60-40 mixture of isomeric product $\mathbf{4 t}$ (major) and $\mathbf{4 c}$ (minor) was prepared by dehydration of $\mathbf{5}$ using catalytic amount of sulfuric acid at $80-85^{\circ}$ with overall $55-65 \%$ yield. The compound $\mathbf{4 t}$ (trans isomer) was also synthesized with $30-35 \%$ by elimination in basic conditions. Both $\mathbf{4 c}$ and $\mathbf{4 t}$ isomers are relatively unstable. The pure $\mathbf{4 t}$ was separated by flash chromatography (b.p; 70-3\%/23 torr).

\section{4t Isomer}

${ }^{1} \mathrm{H}$ NMR $\left(200 \mathrm{MHz}, \mathrm{CDCl}_{3}\right): 0.1-0.9(4 \mathrm{H}), 1.70(1 \mathrm{H}), 4.93$ $(1 \mathrm{H}$, qdd 1,7, 16, H-2), $5.48(1 \mathrm{H}, \mathrm{dqd}, 1,7,16, \mathrm{H}-1), 1.64(3 \mathrm{H}$, dd, $\left.\mathrm{CH}_{3}\right)$

$4 \mathbf{c}$ isomer (spectral data extracted from the $300 \mathrm{MHz}$ NMR spectrum of this mixture).

NMR ${ }^{1} \mathrm{H}\left(300 \mathrm{MHz}, \mathrm{CDCl}_{3}\right)$ : 0.1-0.9 (m, unresolved 4H), 1.3 $(1 \mathrm{H}), 4.70$ (qdd, $1 \mathrm{H}, 10,10,1.8, \mathrm{H}-3), 5.35(1 \mathrm{H}, \mathrm{m}, \mathrm{dqd}, 1,10$, 7, $\mathrm{H}-2), 1.70\left(3 \mathrm{H}, 7,2, \mathrm{CH}_{3}\right)$.

\section{Bicyclopropyl (2)}

This compound was synthesized by three methods.

a. according to the Farneth methodology (28-9) using a Simmons-Smith reaction on a tetrabromo derivative obtained from dibromocarbene addition to butadiene (31) with max. $20 \%$ yield. Compound 2 was used as such in the Diels-Alder reactions.

b. direct coupling of cyclopropylmagnesium bromide to cyclopropylbromide at $-78^{\circ}$ using a Grignard reaction in THF solution with low yield of product 2 (10-2\%). The unsaturated cyclic products and cyclopropanol derivatives were obtained instead in these reactions.

c. dicyclopropyl zinc addition to cyclopropyl bromide at $-78^{\circ}$; yield of 2 within the $5 \%$ range.

The analytical sample of 2 : bp $50-65{ }^{\circ} \mathrm{C}$, one gas-chromatographic peak.

${ }^{1} \mathrm{H}$ NMR (200 MHz, $\left.\mathrm{CDCl}_{3}\right): 0.1(4 \mathrm{H}), 0.3-0.35(4 \mathrm{H})$, $0.80-0.85(2 \mathrm{H})$.

\section{Diels-Alder synthesis with diene precursors (2-8)}

The above mentioned reactions, according to routes a-d, were performed at $50^{\circ} \mathrm{C}$ under thermal conditions in closed stainless steel vessels or in all-glass closed system at room-temperature under the MW irradiation using MAP technology (21-3) and a 
one-pot method with the dienophile present in the vessel. The volatility of the dienes and cylopropane containing intermediates (e.g. 5) require the reaction mixtures, after the quenching with the maleic anhydride to obtain the adducts, to be immediately extracted with ether and analysed by GC-MS and their NMR spectra were recorded. This method was developed in order to separate any polymer fraction due to diene polymerisation, which can be important especially at higher $\left(50{ }^{\circ} \mathrm{C}\right)$ temperatures for all dienes and their precursors. This also minimizes the aromatisation of adducts with or without dienophile expulsion already observed.

After several unsuccessful attempts the thermal reaction temperature was decreased to $25^{\circ}$ and then to $0^{\circ}$, because of the volatile and unstable character of dienes at $50^{\circ}$ (32).

The crude reaction mixtures were analysed by GC-MS. At higher temperatures, reactions with anhydride showed, using GC-MS as a detection method, the presence of up to 40 compounds. These same reactions carried out in a micro-wave assisted mode reduced this number to 25 or 28 . The major adduct diene - anhydride was always present in all these series, and its abundance was used to quantify the data (Table 2).

The use of a closed vessel "one-pot" method conforms to the practice widely applied in combinatorial chemistry for soft reaction conditions in particular. The lower temperatures helped minimizes losses in the volatile substrates and reduced their rearrangements (33).

\section{Synthesis of Diels -Alder adducts.}

Physical description: adducts 14-17 were obtained as oils or semisolid products decomposing easily when heated and their further crystallisation was not attempted. The NMR spectra of adducts 14-17, 22 and 23 were recorded on $500 \mathrm{MHz}$ spectrometer.

\section{Adduct 14 (on 11tt)}

Alternative name: $4 \alpha, 7 \alpha$-dimethyl-3a,4,7,7a-tetrahydro isobenzofurane-1,3-dione (14)

Product 14 was synthesized with a $90-95 \%$ yield.

${ }^{1} \mathrm{H} \mathrm{NMR}\left(\mathrm{CDCl}_{3}\right): 3.34(\mathrm{H}-3 \mathrm{a}$ and $\mathrm{H}-7 \mathrm{a}), 2.48(\mathrm{H}-4$ and $\mathrm{H}-7), 5.77(\mathrm{H}-5$ and $\mathrm{H}-6), 1.43\left(\mathrm{CH}_{3}-\mathrm{C} 4\right.$ and $\left.\mathrm{CH}_{3}-\mathrm{C} 7\right)$

${ }^{13} \mathrm{C}$ : 46.15 (C3a and $\left.\mathrm{C} 7 \mathrm{a}\right), 30.0$ (C4 and $\left.\mathrm{C} 7\right), 134.15$ (C-5 and $\mathrm{C}-6), 16.1\left(\mathrm{CH}_{3}-\mathrm{C} 4\right.$ and $\left.\mathrm{CH}_{3}-\mathrm{C} 7 \mathrm{a}\right), 171.5(\mathrm{C}=\mathrm{O})$.

\section{Adduct 15 (on 11 ct)}

Alternative name: $4 \alpha, 7 \alpha$-dimethyl-3a,4,7,7a-tetrahydro isobenzofurane-1,3-dione (15)

${ }^{1} \mathrm{H}$ NMR $\left(\mathrm{CDCl}_{3}\right) 1.46\left(\mathrm{CH}_{3}-\mathrm{C} 4, \mathrm{CH}_{3}-\mathrm{C} 7\right), 5.85$ and $5.80(\mathrm{H}-$ 5, H-6), 3.28 and 3.41 (H-3a, H-7a), 2.34 and $2.28\left(\mathrm{CH}-\mathrm{CH}_{3}\right)$

\section{Adduct 16 (on 12)}

Alternative name: 4,4-dimethyl-3a,4,7,7a-isobenzofurane-1,3dione (16)
${ }^{1} \mathrm{H}$ NMR $\left(\mathrm{CDCl}_{3}\right)$ :5.6-5.7 (2H, H-5, H-6), $1.23\left(3 \mathrm{H}, \mathrm{s}, \mathrm{CH}_{3}-\right.$ $\mathrm{C} 4 \mathrm{ax})$ and $1.08\left(3 \mathrm{H}, \mathrm{s}, \mathrm{CH}_{3}-\mathrm{C} 4 \mathrm{eq}\right), 3.07(1 \mathrm{H}$, ddd 8.5, 9, 4, H-7a), $2.98(1 \mathrm{H}, \mathrm{d}, 8.5, \mathrm{H}-4 \mathrm{a}), 2.05(1 \mathrm{H}, \mathrm{H}-7 \mathrm{eq})$ and $2.28(1 \mathrm{H}$, H-7ax)

\section{Adduct 17 (on 13)}

Alternative name: 5,6-dimethyl-3a,4,7,7a-tertahydro isobenzofurane-1,3-dione (17)

${ }^{1} \mathrm{H}$ NMR $\left(\mathrm{CDCl}_{3}\right): 3.36$ (tt, 3.5, 1.0; H3a, H7a), 2.46 (td, $-15,1.0, \mathrm{H}-4$ and H-7), 2.29 (broad d, H-4' and H-7'), 1.717 $\left(\mathrm{CH}_{3}-\mathrm{C} 5\right.$ and $\left.\mathrm{CH}_{3}-\mathrm{C} 6\right)$

${ }^{13} \mathrm{C}$ NMR: 40.3 (C3a and C7a), 30.4 (C4 and C7), 132.7 (C-5 and C-6), 174.46 (C1 and C3), CH3 17.6.

Adduct on terpinene (22)

Alternative name: 1 -isopropyl- $\alpha 7$-methyl- $\alpha-4$-oxa -tricyclo[5,2,2,0] 204,204 ] undec-8-ene-3,5-dione (22)

The equimolar quantities of terpenes and maleic anhydride were dissolved in toluene and heated overnight. Adduct $\mathbf{2 2}$ was separated by precipitation after partial removal of the solvent. Yield: $67 \%$.

${ }^{1} \mathrm{H}$ NMR $\left(\mathrm{CDCl}_{3}\right): 3.22(1 \mathrm{H}, \mathrm{d}, 9, \mathrm{H}-2), 2.86(1 \mathrm{H}, \mathrm{d}, 9$, H-6), $6.02(1 \mathrm{H}, \mathrm{d}, 9, \mathrm{H}-8), 6.08(1 \mathrm{H}, \mathrm{d}, 8.5, \mathrm{H}-9), 1,45$ and $1,35\left(1 \mathrm{H}\right.$ each, ddd, 3,16 and 12 and $2.5,11$ and $12 \mathrm{~Hz}, \mathrm{CH}_{2}-$ 10), 1.50 and 1.30 (1H each, 4,12 and 12 ddd, $\left.\mathrm{CH}_{2}-11\right), 1.49$ $\left(\mathrm{CH}_{3}-12\right), 2.547$ (heptuplet, $\left.1 \mathrm{H}, 7, \mathrm{H}-13\right), 1.0\left(3 \mathrm{H}, \mathrm{d}, 7, \mathrm{CH}_{3}-\right.$ 13), $1.08\left(3 \mathrm{H}, \mathrm{d}, 7, \mathrm{CH}_{3}-15\right)$.

${ }^{13} \mathrm{C}$ NMR: 43.4 (C-1), 47.1 (C-2), 170.8 (C-3), 171.4 (C-5), 50.9 (C-6), 36.3(C-7), 136.9 (C-8), 136.2 (C-9), 22.6 (C-10), 33.5 (C-11), 22.1(C-12), isopropyl group 29.3 (C-13), 18.2(C-14), 16.6 (C-15)

Adduct of $\alpha$-phellandrene (23)

Similar procedure to adduct 22. Yield: $77 \%$

${ }^{1} \mathrm{H}$ NMR $\left(\mathrm{CDCl}_{3}\right): 3.18(1 \mathrm{H}$, ddd, 2.0, 3.5 and $6.0, \mathrm{H}-1)$, $3.08(1 \mathrm{H}, \mathrm{dd}, 3.5$ and $8.5, \mathrm{H}-2), 3.14(1 \mathrm{H}$, dd 3.5 and $8.5, \mathrm{H}-$ 6), $2.96(1 \mathrm{H}$, dddd, $0.5,2.0,3.0$ and 5.0, H-7), $1.32(1 \mathrm{H}, \mathrm{m}$, 1.5, 5.5, 7.0 and 9.0, H-10), 1.79 and $1.044(2 \mathrm{H}$, ddd, 3.0, 9.0 and 3.5; ddd 3.5, 8.5 and 3.5, $\mathrm{H}-11$ ax and $\mathrm{H}-11 \mathrm{eq}), 1.78$ (3H, $\mathrm{d}, 2.0, \mathrm{H}-12)$, isopropyl chain $1.12(1 \mathrm{H}, \mathrm{d}$, heptuplet, 9.5 and 7.0 , H-13), 0.92 (3H, d, 7.0, CH14), 0.81(3H,d, 7.0, $\left.\mathrm{CH}_{3}-15\right)$, $5.76(1 \mathrm{H}, \mathrm{m}, \mathrm{dqd}, 0.5,2.0,6.5(\mathrm{H}-9))$

${ }^{13} \mathrm{C}$ NMR: 35.06 (C-1), 46.07 (C-2), 173.0 (C-3), 172.7 (C-5), 44.04 (C-6), 37.72 (C-7), 142.2 (C-8), 122.3 (C-9), 44.27 (C-10), 29.7 (C-11), 20.43 (C-12), 32.86 (C-13), 20.7 (C-14), 20.12 (C-15)

\section{References}

1. Alder, K.; Stein, G. Angew. Chem. 1937, 50, 510

2. Woodward, R.B.; Hoffmann, R. "Conservation of orbital symmetry”, Verlag chemie, Weiheim, (1970); Woodward, R.B.; 
Hoffmann, R. J. Amer. Chem. Soc. 87, (1965) 395; Woodward, R.B.; Hoffmann, R. Angew. Chem. Int. Ed. 1969, 8, 781

3. Kloetzel, M.C.; Org. Reactions, 4, (1948) 1; Holmes, H.L.; Org. Reactions, 4, (1948) 60; Butz, L.W. and Rytina, A.W.; Org. reactions, 5, (1949) 136; Martin, J.G.; Hill, R.K.; Chem. Rev. 61, (1961) 537; Diels, O.; Alder, K. Ann., 460, (1928) 98; Diels, O.; Alder, K. Ann., 470, (1929) 62; Diels, O.; Alder, K.; Ber., 62, (1929) 2081; Diels, O., Alder, K.; Ber., 62, (1929) 2087; Littmann, E.R. Ind. Eng. Chem., 28, (1936) 11509

4. Corno, C.; Ferraris, G.; Priola, A.; Cesca, S. Macromolecules 1979, 12, 404

5. Cesca, S. "Isomerisation Polymerisation" Encyclopedia of Polymer Science and Engineering, Vol. 2, J,Wiley, New York, 1985

6. Jankowski, C.K.; Dako, E.; Boulaouz, A.; Delaforge, M.; Paré, J.J.R.; Bélanger, J.M.R. Spectroscopy 2007, 12, 293

7. Snyder, L.C.; Meiboom, S. J. Amer. Chem. Soc. 1967, 89, 1038; Pelletier, A.; M.Sc, Thesis, Université de Moncton, 2008

8. Charton, M. and Zabicky, J.; "The chemistry of alkenes" Interscience, NewYork, 2, (1970) 511; Staley, S.W.; J. Amer. Chem. Soc. 1967, 89, 1532; Pews, R.G. and Ojha, N.D.; J. Amer. Chem. Soc. 1969, 91, 5769

9. Fleming, I.; Frontier Orbitals and Organic Chemical Reactions, J.Wiley, New York, $7^{\text {th }}$ ed., (1976)

10. Bartlett, P.D. Tetrahedron 1980, 36, 2; Rhoads, S.J.; and Raulins, N.R. Org. Reactions 22, (1975) 1

11. Baldwin, J. Chem. Rev. 2003, 103, 1197 and references quoted therein

12. Overton, K.H.; Specialist Periodical Report: Terpenoids and steroids, Vol. 4, 42-6; Cooper, M.A.; Holden, C.D.; Loftus, P.; Whittaker, D. J. S. C. Perkin II, 1973, 665

13. However it is worth noticing that the sigmatropic shift was considered by Woodward-Hoffmann formalism only for a limited number of concerted reactions. The vinylcyclopropane (1) system could undergo a sigmatropic shift only after allylic rearrangement and followed by the opening of the cyclopropane ring. The allylic and/or sigmatropic shifts can then transform the vinylcyclopropane or bicyclopropyl into isomeric dienes of specific geometry. The Cope rearrangement can also be considered as a step toward the final conjugated diene formation. The vinylcyclopropane to cyclopentene rearrangement could then be seen as a 1,3-sigmatropic reaction. The accepted mechanism of this reaction proceeds via the formation of 1,5-diradicals in two isomeric configuration: $\mathrm{Z}$ (cis) or $\mathrm{E}$ (trans). The first one leads to cyclopentene and the second to dienes or other products (Scheme 4).

14. Jankowski, C.K.; Pelletier, A.; Diaz, E.T.; Bélanger, J.M.R.; Paré, J.J.R.; Aumelas, A.; Besson, T.; Pereira ,M.de F.; Mauclaire, L. Can. J. Chem. 2007, 85, 996; Jankowski, C.K.; Savoie, A.; Lesage, D.; Paré, J.J.R.; Bélanger, J.M.R.; Pereira, M.de F.; Thierry, V.; Besson, T.; Maciejak, O.; Toma, F.; Mauclaire, L.; Lia, X. J. Mex. Chem. Soc. 2006, 50, 90.

15. Elis, R.J.; Frey, H.M. Proc. Chem. Soc. 1964, 221; Roth, W.R. and Konig, J.; Ann., 688, (1965) 28; Ohloff, G.; Tetrahedron Lett. 1965, 3795; Jorgenson, M.J.; Thacher A.F. Tetrahedron Lett. 1969, 4651; Corey, E.; Yamamoto, Y.; Herron J.T.; Achiwa, K. J. Amer. Chem. Soc. 1970, 92, 6635; Dolbier, R.; Sellers, S.F. J. Org. Chem. 1982, 47, 1
16. Hirsch, J.A. Concept in Theoretical Organic Chemistry, Allyn and Bacon, Boston, (1974) 79-83

17. The parent of these terpenes should be considered thujene (9) with a homoannular and endocyclic vinylcyclopropane which unfortunately is not commercially or easily available as a pure isomer.

18. Diels, O.; Koch, W.; Frost, H. Ber. 1938, 71, 1163

19. Goodway, N.F. and West, T.F.; Nature 1937, 934

20. Overton, K.H., Ed.; Specialist Periodical Report: Terpenoids and Steroids, Vol.4, The Chemical Society, Burlington House, London, 1974, 42-46.

21. Kasali, A.A.; Ekundayo, O.; Paul, C.; Konig, W.A.; Phytochemistry, 2002, 59, 805; Bulow, N.; Konig, W.A. Phytochemistry 2000, 55, 141

22. Katritzky, A.R.; Cai, C.; Collins, M.D.; Scriven, E.F.V.; Sing, S.K. J. Chem. Ed., 2006, 83, 634-636; Bougrin, K.; A.Loupy and M.Souiaoui, I.Photochem.Photobio.C Photochemistry Reviews, 6 (2-3) 139-67 (2005); Hoz, A.de la; Diaz-Ortiz, A.; Moreno, A.; Critical Rev. 2005, 34, 164-178 See also the references quoted therein; Kappe, C.O.; Angew. Chem. Int. Ed. 2004, 43, 6250-6284.

23. Bélanger, J.M.R.; Alfaro, M.J.; Padilla, F.C.; Paré, J.R.J. Food Research International, 2003, 36, 499-504; Paré, J.R.J.; Rochas, J.-F.; Jacomino, J.-M.; Sanchez, F.N.L.; Bélanger, J.M.R.; Proceedings of the International Symposium on Microwave Science and its Application to Related Fields, Takamatsu, Japan, 2004, 25-28; Paré, J.R.J.; Bélanger, J.M.R.; AMPERE Newsletter, 2006, $51,1-4$

24. Paré, J.R.J.; Bélanger, J.M.R.; Punt, M.M.; US Patent 6,061,926 (2000)

25. Reetz, M.T.; Raguse, B.; Marth, C.F.; Huegel, H.M.; Bach, T.; Fox, D.N.A. Tetrahedron 1992, 48, 5731; Shibata, T.; Tabira, H.; Kenso, S.; J. Chem. Soc. Perkin Trans. 1998, 177.

26. Several attempted synthesis described in the literature mentioned the assumed presence of these products, their short lifespan, easy polymerisation or aromatisation. Several procedures were either incomplete or irreproducible. Many particular and non commercial reagents are used in order to obtain e.g. the bicyclopropane (29), in particular zinc-Grignard $(24,28)$ or photolysis reactions (27).

27. Julia, M. Bull. Soc. Chim. France 1961, 1849.

28. Khusid, A.K. J. Org. Chem. USSR, 1987, 23, 112 (English translation)

29. Farneth, W.E.; Thomsen, M.W.; J. Amer. Chem. Soc. 1983, 105, $1843-1848$

30. Ferreri, C.; Ambrosone, M.; Chatgilialoglu, C. Synth. Commun. 1995, 25, 3351.

31. Landgeber, J.A.; Becker, L.W.; J. Amer. Chem. Soc. 1968, 90, 395.

32. The above mentioned difficulties are related to the six carbon or lower chains of dienes involved and to the cyclopropylcarbinols. The other isomeric model $\mathrm{C}_{6}$ dienes were not commercially accessible. Some of them cannot be obtained from the cyclopropane compounds of this study and then are not potential diene precursors.

33. The small volume stainless steel vessel conditions are relatively close to those applied in using the MW methods. 